\title{
DIE FREIHEITSKONZESSION DES LANDGRAFEN VON HESSEN-KASSEL, DAS EDIKT VON POTSDAM UND DIE ANSIEDLUNG VON HUGENOTTEN IN BRANDENBURG-PREUSSEN UND HESSEN-KASSEL
}

Die Einwanderungs- und Ansiedlungspolitik Brandenburg-Preußens und Hessen-Kassels, also zweier deutscher Staaten, die in der Forschung als die bekanntesten Länder des Refuge im Alten Reich gelten, darf in einem Band zu Immigrationspolitik deutscher Staaten und Integrationsprozessen französischer Kalvinisten in der Frühen Neuzeit nicht fehlen. Diese beiden Staaten nahmen im Alten Reich nach 1685 die meisten französischen Protestanten auf. Die Forschung geht davon aus, daß sich in Hessen-Kassel nach 1685 ca. 3800 französische Flüchtlinge, in Brandenburg-Preußen ca. 20000 Refugiés niederließen ${ }^{1}$. So sehr sich die Immigrationspolitik der einzelnen europäischen Staaten in Bezug auf die Refugiés in manchen Elementen glich bzw. konzertierte Hilfsaktionen und eine gesamteuropäische Lösung für das Flüchtlingsproblem angestrebt wurden, wie dies unter anderem die Korrespondenz zwischen der Schaltzentrale der Refugiés in Genf und Lausanne und den protestantischen Regierungen in Europa eindrücklich belegt ${ }^{2}$, so sehr zeigen die Beispiele Hessen-Kassel und Brandenburg-Preußen, daß die Immigrationspolitik immer auch von persönlichen Präferenzen des Herrschers bzw. den spezifischen politischen und wirtschaftlichen Interessen der Aufnahmeländer bestimmt war.

\section{Die Landgrafschaft Hessen-Kassel}

Die Kontakte zwischen den Landgrafen von Hessen-Kassel, die 1605 zum Kalvinismus übergetreten waren, und der Reformierten Kirche in Frankreich gehen auf die 1550er Jahre zurück. Nicht nur unterstützten Truppen des Landgrafen Philipp von Hessen-Kassel das kalvinistische Lager während der Glau-

\footnotetext{
${ }^{1}$ Robin D. GWYNN, Huguenot Heritage. The history and contribution of the Huguenots in Britain, London, New York ${ }^{2} 1988$, S. 24.

${ }^{2}$ Vgl. die Kopien der Papiers Antoine Court in der Bibliothèque de la Société de l'histoire du protestantisme français, Paris (= BibSHPF).
} 
benskriege in Frankreich (1560-1598), auch der intellektuelle Austausch zwischen der von Landgraf Philipp gegründeten Universität in Marburg und Vertretern der protestantischen Akademien in Saumur, Montpellier und Genf blickte 1685 bereits auf eine fast 130jährige Geschichte zurück. Die ersten meist wallonischen Flüchtlinge wurden zu Beginn des 17. Jahrhunderts in Hessen-Kassel unter Landgraf Moritz (1592-1627) zur Hebung von Handel und Gewerbe angesiedelt.

Insbesondere Henri de Mirmand ${ }^{3}$ gelang es, Landgraf Karl (1670-1730) für die zunehmenden Verfolgungen der Protestanten in Frankreich ab den 1660er Jahren zu sensibilisieren. So verwundert es kaum, daß im Mai 1680 François de Paule, Chevalier, Seigneur de Sardan, Vicomte de la Houssaye, dem Landgrafen ein Mémoire präsentierte, in dem er und seine Glaubensgenossen die Etablierung von französisch-kalvinistischen Colonien in Hessen-Kassel forderten. Doch noch 1680 verweigerte der Landesherr französischen Protestanten die angemahnten Privilegien.

Erst am 18. April 1685 bewilligte der Landgraf die Freyheits-Concession und Begnadigung ${ }^{4}$, die noch von vor der Revokation des Edikts von Nantes datiert. Auf sie folgten dann am 1. August 1685 weitere Privilegien und am 12. Dezember 1685 die Concessions et Privileges ${ }^{5}$.

Trotz der bestehenden engen Kontakte zwischen Herrscherhaus und französischen Reformierten in Frankreich und in der Schweiz lud die FreyheitsConcession nicht explizit französische Kalvinisten nach Hessen-Kassel ein, sondern wandte sich an alle Reformierten, die bereit waren, in Hessen-Kassel Manufakturen zu errichten oder Gewerbe und Handel zu treiben. Die Privilegien enthielten im April 1685 folgende Garantien: Den reformierten Einwanderern wurden 1. der besondere Schutz des Landgrafen, 2. die Möglichkeit des Land- und Hauserwerbs in ganz Hessen-Kassel mit dem Ziel der Neubebauung von Wüstungen, 3. Zunftfreiheit, 4. zehn Jahre Abgabenfreiheit sowie Freiheit von Einquartierungen und Diensten und 5. Zollfreiheit innerhalb Hessen-Kassels zugestanden. Voraussetzung für diese Privilegien war, daß die Immigranten Ihro HochFürstl. Durchl. Gesetzen beschriebenen gemeinen Rechten und andern außgelassenen Ordnungen sich gemäß verhalten/ und darjegen nicht muthwillig freveln, d.h. Gesetzestreue und Unterordnung unter die Ordnung und Regeln des Landes.

\footnotetext{
${ }^{3}$ Henri Marquis de Mirmand, ein aus Nîmes stammender reformierter Adliger, gehörte in der Schweiz zu den wichtigsten Organisatoren der Flüchtlingshilfe nach 1685.

${ }^{4}$ Im folgenden wird auf die Fassung der Freyheits-Concession und Begnadigung zurückgegriffen, wie sie als Kopie in deutscher Sprache in der BibSHPF (wie Anm. 2), vorliegt. Vgl. ibid., Mss. 780-1 und -2.

${ }^{3}$ Franz-Anton KADELL, Die Hugenotten in Hessen-Kassel, Darmstadt, Marburg 1980, S. 296.

${ }^{6}$ BibSHPF (wie Anm. 2), Mss. 780-1 und -2 .
} 
Unter dem Einfluß, den sich Jean Feuquier am Kasseler Hof zu verschaffen verstand, wurden dann jedoch im August 1685 weitere Privilegien erlassen. Bezüglich des französisch-reformierten Glaubens wurde nun den Refugiés finanzielle Hilfe beim Unterhalt ihrer Kirchen, Pastoren und Schulmeister zugesichert. Die Freijahre wurden von zehn auf zwölf erhöht. Dazu kam, daß die immigrierenden Meister und Gesellen nun unter der persönlichen Kontrolle des Landgrafen standen, dem sie einen Treueid - in französischer Sprache zu leisten hatten. Rechtlich wurde ihnen eine exemte Stellung eingeräumt, die sie zunächst von kommunalen Institutionen weitgehend unabhängig machte.

Ziel Feuquiers und seiner Glaubensgenossen war gerade diese exemte wirtschaftliche und rechtliche Stellung der Refugiés, ein Ziel, das auch in Brandenburg-Preußen und in Irland angestrebt werden sollte. Doch diese Ziele entsprachen eigentlich nicht den Wünschen des Landgrafen, der die Refugiés im Bereich der Jurisdiktion und in ökonomischer Hinsicht seinen Untertanen gleich-, nicht besserstellen wollte. Die sogenannte Chancellerie française, die aus der 1685/86 gegründeten Flüchtlings- und Ansiedlungskommission hervorgegangen war, war jedoch eine Institution nach dem Willen Feuquiers. Sie allein wurde mit den Angelegenheiten der Refugiés, auch in Rechtssachen, betraut. In ihrer Rechtsprechung hatte sie sich jedoch strikt nach Landesrecht und hessischem Gewohnheitsrecht zu richten. Direktor der Kanzlei war von Anfang an ein Deutscher, dem dann 1686 Refugiés als commissaires bzw. conseillers beigeordnet wurden. Leiter der Chancellerie française war Regierungsrat von Halcke, den 1689 von Rochau ablöste. 1686 wurde Jean Baptiste Feuquière zum französischen Sekretär der Kanzlei ernannt. Ihm folgten während der Regierungszeit Landgraf Karls weitere Refugiés. Conseiller noble de la Sérénité de Hesse et Commissaire pour les affaires des françois wurde im März 1686 ebenfalls ein Franzose: Frédéric de Lalouette, Chevalier de Vernicourt. Er wurde für die Kasseler Refugiés zuständig, während Pierre Daubigny Bevollmächtigter für die Landstädte und Dörfer der Refugiés war. Beide übten auch richterliche Funktionen aus. Zur Zeit Landgraf Friedrichs II. (1720-1785, Landgraf seit 1760) waren dann bereits alle leitenden Mitglieder der Chancellerie française Deutsche ${ }^{7}$.

Den Höhepunkt der Bemühungen Feuquiers um herausragende Privilegien für die Refugiés in Hessen-Kassel bildeten die Concessions et Privileges vom 12. Dezember 1685, die sich explizit darum bemühten, à satisfaire les demandes et les privileges demandez seitens Feuquiers und seiner Klienten. Nachdem bereits am 1. Dezember 1685 ein Spendenaufruf an die Untertanen des Landes mit der Aufforderung ergangen war, den genötigten armen Leuten und Glau-

${ }^{7}$ KADELL, Hugenotten (wie Anm. 5), S. 345. 
bensgenossen so viel möglich/ unter die Armen zu greiffen (auß Christlicher compassion resolviret) ${ }^{8}$, folgten dann elf Tage später weitere Privilegien.

Nun, da nach dem Erlaß des Edikts von Fontainebleau der Kampf der protestantischen Staaten Europas um die besten Refugiés entbrannt war, fing auch das bisher doch eher zurückhaltende Hessen-Kassel an, um die Refugiés zu sbuhlen. Nicht nur wurde nun Werbung für das Land gemacht, sondern es wurden auch zusätzlich zu den kalvinistischen Manufakturisten rentiers, die ihr Vermögen im Land zu Zinsen anlegen sollten, eingeladen, sich in der Landgrafschaft anzusiedeln. Ziel der Ansiedlung war es trotzdem weiterhin vor allem, das Land mit ihrer Hilfe zu bereichern.

So sehr der Landgraf für eine rechtliche und wirtschaftliche Gleichstellung von Refugiés und deutschen Untertanen optierte und zunächst einen Kompromiß schließen mußte, so sehr war er trotzdem gegen eine Vermischung der beiden nations. Die Refugiés, so der Plan, sollten Wüstungen neu besiedeln und in neu gegründeten Städten Wirtschaft und Handel in Schwung bringen. Doch die Stadtverwaltung von französischen Städten wie Sieburg, später Karlshafen, wurde bereits zu Beginn des 18. Jahrhunderts paritätisch mit "Deutschen« und "Franzosen « besetzt. Zumindest in den neugegründeten Städten sowie auch in der Oberneustadt Kassel mischten sich Deutsche und Franzosen schnell'.

Das Interesse Karls an der Ansiedlung von Refugiés war nicht von Dauer. Bereits 1688, angesichts eines erneuten Flüchtlingsstroms aus Zürich und Bern, kam es zu einer Weigerung Landgraf Karls, weitere französische Immigranten in sein Land zu lassen ${ }^{10}$. Erst 1698 wurden erneut mit den Hersfelder Privilegien, in vielen Punkten eine Bestätigung der Concessions et Privileges, französische Protestanten angesiedelt: Mons. le landgrave de Hesse[-Cassel] veut bien, à ce qu'on dit, donner encore des terres à ceux qui voudraient en aller défricher dans ses états, hieß es in einem Schreiben Mirmands vom September $1698^{11}$. Doch auch dieses Privilegium war kein Toleranzedikt und gestattete die Immigration und Ansiedlung von Refugiés nur in begrenztem Umfang. Ihre rechtliche Sonderstellung wurde beibehalten.

Unter den Nachfolgern Landgraf Karls kam es verstärkt zu Problemen bei der Erneuerung der Privilegien, wie sie bei Regierungsantritt eigentlich üblich gewesen wäre. 1731 bestätigte Landgraf Friedrich I., gleichzeitig König von Schweden, zwar die Concessions et Privileges, sorgte aber dafür, daß Zunftund Gewerbeangelegenheiten unter seine direkte Kontrolle kamen ${ }^{12}$.

\footnotetext{
${ }^{8}$ Siehe Kopie des Aufrufs in BibSHPF (wie Anm. 2), Mss. 780-1 und -2.

${ }^{9}$ KADELL, Hugenotten (wie Anm. 5), S. 254f., 626.

${ }^{10}$ Ibid., S. 25.

"BibSHPF (wie Anm. 2), Papiers Court, Copies, Ms. 618 AA.

${ }^{12}$ KADELL, Hugenotten (wie Anm. 5), S. 339-341.
} 
1751, nach dem Tod Friedrichs I., mußten die französischen Protestanten 14 Jahre warten, bis ihnen dann doch die Privilegien von 1685 erneut bestätigt wurden. Zwar wurden diese noch einmal 1786 emeuert, doch hatte die Regierung der Landgrafschaft in der Zwischenzeit dafür gesorgt, daß die französischen Colonien in Hessen-Kassel unter der administrativen, judikativen und wirtschaftlichen Kontrolle der allgemeinen Institutionen des Territoriums standen. Gegen Ende des 18. Jahrhunderts war damit in Hessen-Kassel der privilegierte Status der französischen Protestanten zwar de iure noch vorhanden, de facto jedoch schon weitgehend unterminiert worden. Diesem Prozeß trugen dann die offizielle Unterstellung aller französischen Colonien unter die deutsche Amtsobrigkeit 1800 und 1804 die Auflösung der Chancellerie française Rechnung.

\section{Brandenburg-Preußen}

Was Brandenburg-Preußen von vielen anderen nominell protestantischen Staaten Europas - außer Irland - gegen Ende des 17. Jahrhunderts unterschied, war die Wahrnehmung dieses Landes als zutiefst rückschrittlich und unterentwikkelt. Diese Wahrnehmung teilte die Regierung Brandenburg-Preußens nicht nur mit den Regierungen und der öffentlichen Meinung anderer europäischer Staaten, sondern vor allem auch mit vielen französisch-reformierten Diploma$\operatorname{ten}^{13}$. Große Zugeständnisse an die Einwanderungswilligen waren demnach programmiert.

Ähnlich wie die Landgrafen von Hessen-Kassel hatten auch die Kurfürsten von Brandenburg zu Beginn des 17. Jahrhunderts, nämlich 1613, den kalvinistischen Glauben angenommen. Die Mehrheit der Bevölkerung blieb lutherisch.

Für die Zeit zwischen 1645 und 1685 sind die ersten, allerdings noch sporadischen, Ansiedlungsversuche von Refugiés in Brandenburg-Preußen zu verzeichnen. Eine einheitliche Immigrations- und Privilegienpolitik, d.h. eine allgemein verbindliche Rechtsgrundlage für die Einwanderung und die Ansiedlung von französisch-reformierten Protestanten, fehlte bis 1685. Auffallend sind die wiederholten Versuche der 1672 etablierten Französisch-Reformierten Kirche Berlins, Einfluß auf Ansiedlungsbedingungen und Privilegien für die sich um den Hof des Kurfürsten entwickelnde französische Colonie zu nehmen. Die Flüchtlinge präsentierten sich als selbstbewußte Partner des Kurfürsten und verstanden es, ihm Privilegien, vor allem in Kirchensachen, abzu-

${ }^{13}$ Stefi JERSCH-WENZEL, Juden und "Franzosen« in der Wirtschaft des Raumes Berlin/Brandenburg zur Zeit des Merkantilismus, Berlin 1978, S. 32. 
trotzen, die eigentlich dem erklärten Willen Friedrich Wilhelms zuwiderliefen. Dies wird am Beispiel der Etablierung eines Konsistoriums der französischreformierten Gemeinde und der Einführung der discipline der kalvinistischen Kirche Frankreichs besonders deutlich. Das 1682 gebildete Konsistorium sollte unter der Bedingung gegründet werden, daß es se conformeroit à la discipline du dome [d.h. der Deutsch-Reformierten Kirche], et dans le droit ecclesiastique, qu'elle auroit le grand consistoire allemand pour juge. Darüber hinaus sollte es sich selbst in der Öffentlichkeit weder als Konsistorium bezeichnen noch die "Ältesten« als solche ausweisen. Unter dem Dach der DeutschReformierten Kirche wurde der Französisch-Reformierten Kirche mit der Etablierung eines eigenen Konsistoriums damit eine gewisse Autonomie zugestanden, die allerdings nach dem Willen des Kurfürsten und seiner Berater nicht publik werden durfte. Bis 1684 wurde dieser Kompromiß offiziell von beiden Seiten eingehalten. Dann jedoch forderte die französisch-reformierte Gemeinde die öffentliche Anerkennung ihrer Eigenständigkeit und das Recht, Mitglieder der Gemeinde gemäß dem Kirchenrecht für unmoralisches Verhalten abstrafen und auch offiziell als Konsistorium auftreten zu können. Dieser Bitte entsprach der Kurfürst und erlaubte der Gemeinde nun sogar

d'élire des anciens, devant lesquels assistés d'un des Ministres de la cour, les affaires qui concernent une bonne discipline soient, selon la coutume de France, évoquées et terminées; Que donc chaque membre de cette assemblée se règle et se soumette d̀ cette ordonnance, comparoisse devant ses Pasteurs et anciens, quand il y sera cité, et obeisse selon son devoir à leurs décrets et réglemens ${ }^{14}$.

Die französisch-reformierte Gemeinde durfte sich also bereits vor Erlaß des Edikts von Potsdam auf Betreiben ihrer Ältesten als eigenständige Gemeinde, ausgestattet mit der "Discipline ecclésiastique» der kalvinistischen Kirche Frankreichs unter der Kontrolle eines Hofbeamten stehend konstituieren.

Am 29. Oktober 1685, gemäß dem gregorianischen Kalender am 8. November 1685, also 22 Tage nach dem Erlaß des Edikts von Fontainebleau (17. Oktober 1685 gemäß dem gregorianischen Kalender), wurden die schon seit den 1660er Jahren gewährten Privilegien für französischreformierte Einwanderer im Edikt von Potsdam erweitert und standardisiert. Bedingung für die Gewährung der Privilegien war, daß sich die Refugiés dauerhaft in Brandenburg-Preußen ansiedelten. Eine Rückkehr nach Frankreich, wie sie sich die Refugiés in England und Irland über Generationen offen hielten, war von Seiten des Großen Kurfürsten Friedrich Wilhelm ebenso wenig vor-

${ }^{14}$ Alle Zitate dieses Abschnittes finden sich in Archiv der Französischen Kirche im Französischen Dom (= AFrD), Registre des Actes consistoriaux de l'Eglise française reformée de Berlin, Bd. 1, 10. Juni 1672 bis 4. Juli 1694, fol. 3r, 10v, 11r, 16v, 18r. 
gesehen wie die Freizügigkeit, die den Refugiés in Hessen-Kassel in den ersten Jahren sogar unter Erlassung des üblichen Abzugsgeldes gewährt worden war ${ }^{15}$.

Auf wirtschaftlichem Gebiet gleichen sich die Privilegien des Dezemberdekrets in Hessen-Kassel und des Edikts von Potsdam sehr. Der Kurfürst gestand zehn bis zwölf Jahre Steuer- und Abgabenfreiheit (ausgenommen die Akzise) sowie Zunftfreiheit zu. Ebenso wurden Vergünstigungen beim Hausbau und -kauf, beim Landerwerb und die Befreiung von Einquartierungen gewährt.

Weiterreichender als die Hessen-Kasseler Privilegien waren die des Edikts von Potsdam bezüglich der Etablierung französisch-reformierter Kirchen in Brandenburg-Preußen. Nominell sollten französisch-reformierte Kirchen gemäß der »Discipline ecclésiastique des églises reformées de France« eingerichtet werden. Der Kurfürst entsprach damit nun auch offiziell den im Vorfeld des Edikts von den Einwanderern abgetrotzten Privilegien, allerdings nur auf Gemeindeebene. Synoden, integraler Bestandteil der kalvinistischen Kirche Frankreichs, wurden untersagt, der Kurfürst mußte als Oberhaupt der Französisch-Reformierten Kirche anerkannt werden, ähnlich wie bereits 1562 in der Pfalz. Damit war die Autonomie der einzelnen Gemeinden der französisch-reformierten Kirchen in Brandenburg-Preußen klar beschnitten. 1701 wurde überdies durch König Friedrich I. ein Oberkonsistorium eingerichtet, um die Kirchenverwaltung der französisch-reformierten Kirchen Brandenburg-Preußens zu vereinheitlichen und die einzelnen Gemeinden kontrollieren zu können ${ }^{16}$. In diesem Oberkonsistorium saßen neben Kommissaren des Königs auch Franzosen. Auch diese Institution widersprach dem Gemeindeprinzip der Französisch-Reformierten Kirche.

Was die französisch-reformierten Colonien in Brandenburg-Preußen jedoch von denen in England, den Niederlanden, Irland, der Schweiz oder HessenKassel am deutlichsten unterschied, war die Etablierung einer eigenen Jurisdiktion in den einzelnen Colonien, für Streitfälle innerhalb der Colonie ${ }^{17}$, die nach französischem Recht, und nicht nach dem jeweiligen Landesrecht entschied. 1685 bereits explizit Bestandteil des Edikts von Potsdam, wurde dieses Privileg 1690 nochmals eindrücklich bestätigt ${ }^{18}$. Da die Refugiés aus unterschiedlichen Regionen Frankreichs mit unterschiedlichen Rechtssystemen und -ordnungen kamen, wurde 1699 für die Refugiés in Brandenburg nicht nur in der Ordonnance française ein für alle Refugiés in Brandenburg-Preußen geltendes Recht geschaffen, das 1702 von König Friedrich I. in Kraft gesetzt

${ }^{15}$ KADELL, Hugenotten (wie Anm. 5), S. 449. In den Anfangsjahren wurde den Kolonisten das Abzugsgeld sogar erlassen.

${ }^{16}$ Christian Otto MYLIUS, Recueil des édits, ordonnances, règlements et rescrits contenant les privilèges et les droits attribués aux françois réfugiés dans les États du Roy de Prusse, Berlin 1750, Sp. 191-194.

${ }^{17}$ Streitfälle zwischen Franzosen und Deutschen wurden von einem französischen Schiedsrichter und dem jeweiligen deutschen Magistrat geregelt.

${ }^{18}$ MYLIUS, Recueil (wie Anm. 16), Sp. 83-86. 
wurde, sondern das Recht innerhalb der französischen Colonien wurde damit auch schneller vereinheitlicht als in allen anderen Teilen BrandenburgPreußens ${ }^{19}$. Hier wurde das "Allgemeine Preußische Landrecht» erst 1794 eingefuihrt.

Analog zum Vorläufer der Chancellerie française in Hessen-Kassel wurde am 23. November 1685 in Berlin eine Kommission unter Leitung des Oberhofmarschalls Joachim Ernst von Grumbkow (ab 1689 unter Ezechiel von Spanheim, ab 1697 unter Eberhard von Danckelmann, dann Alexander Graf von Dohna) gebildet, die sich um die Belange der Refugiés zu kümmern hatte $^{20}$. Dieser später sogenannten Französischen Kommission gehörten auch etliche Refugiés an. 1708 wurde das Französische Kommissariat aus dem Generalkriegskommissariat herausgelöst. Ein sogenannter Departement-Minister vertrat die französischen Colonien direkt im Geheimen Staatsrat. Über diese Funktion waren die Colonien der deutschen Verwaltungshierarchie eingegliedert und unterstanden direkt dem König. Dies versuchte Friedrich Wilhelm I. $1715 \mathrm{zu}$ ändern, allerdings, wie unten zu zeigen sein wird, ohne Erfolg.

Mit diesen Regelungen wurde es den französisch-reformierten Colonien ermöglicht, mit ausdrücklicher Zustimmung des Kurfürsten einen >Staat im Staate zu bilden, dessen einzelne Mitglieder und Institutionen gegenüber den deutschen Untertanen eindeutig privilegiert waren und dessen Rechts- und Kirchenordnung dieser nation einen festeren Zusammenhalt gaben, als dies unter den Untertanen Friedrich Wilhelms in Kleve, Brandenburg oder Preußen bis dato der Fall gewesen war.

1689 wurden dann nochmals für die aus der Pfalz vertriebenen Reformierten, unter ihnen etliche französischer Herkunft, Privilegien zu deren Ansiedlung in Magdeburg gewährt. Sie glichen in vielem denen des Edikts von Potsdam. In einigen Punkten, wie etwa der Bestimmung, die den Neusiedlern 15 Jahre Abgabenfreiheit gewährt, gingen sie sogar über die Privilegien von 1685 hinaus. Ebenso wenig wie 1685 wurde mit diesen Privilegien eine Integration $^{21}$ der Flüchtlinge von Regierungsseite intendiert:

${ }^{19}$ Klaus BRANDENBURG, Die Rechtsprechung in der Kolonie, in: Sibylle BADSTÜBNERGROGER u.a. (Hg.), Hugenotten in Berlin, Berlin 1988, S. $282 \mathrm{f}$.

${ }^{20} 1704$ wurde die Direktion der Französischen Affairen neu geordnet. Eusebius von Brandt, der wegen Abwesenheit von Dohnas die Französische Kommission quasi geleitet hatte, wurde der Rat C.F. von Bartholdi zugewiesen. Gemeinsam hatten sie nun die Aufsicht über die Verwaltung der französischen Colonien und ihrer Kirchengemeinden.

${ }^{21}$ Unter Integration, auch oft synonym mit Amalgamierung gebraucht, wird hier ein Anpassungsproze $\beta$ verstanden, in dem Immigranten Charakteristika des Aufnahmelandes annehmen, ohne daß sie dabei die ursprüngliche Identităt der eigenen Gruppe aufgeben. Der Begriff selbst taucht in den zeitgenössischen Quellen nicht auf. Dennoch ist erwiesen, daß einige der Länder des Refuge wie England, Schweden oder Irland die Integration, avant la lettre, der Flüchtlinge in Religions- und Rechtssachen als Bedingung für deren Aufnahme formulierten und Integration auch bezüglich des Spracherwerbs einforderten. Vgl. Susanne LACHENICHT, Migration, Migrationspolitik und Integration: Hugenotten in Brandenburg- 
Il est permis à cette Colonie demeurante dans ces deux villes voisines de former un corps $\dot{a}$ part \& une communeauté [sic] qui soit \& demeure sous son propre \& particulier Magistrat, \& aura pour jamais le Droit d'avoir des Juges \& Magistrats de leur Religion 22.

Die hohenzollernsche Ansiedlungspolitik erfuhr jedoch spätestens 1692 drastische Veränderungen. Kurfürst Friedrich III. weigerte sich zu Beginn des Jahres 1692, wie aus einem Brief an den englischen König, Wilhelm III., hervorgeht, weitere Flüchtlinge aufzunehmen. Brandenburg-Preußen, so Friedrich III., habe genug für die Refugiés getan, und ihnen unter Garantie von Glaubensfreiheit und öffentlichem Gottesdienst in französischer Sprache Zuflucht gewährt. Nun, 1692, seien jedoch andere protestantische Staaten gefordert, das Flüchtlingsproblem zu lösen ${ }^{23}$. Noch 1698 beschwerte sich de Mirmand, daß sich die reformierten Fürsten im Alten Reich weigerten, weitere Refugiés ins Land zu lassen, und die Organisatoren des Flüchtlingsproblems in der Schweiz deshalb gezwungen seien, lutherische Landesherren von der Aufnahme von französischen Kalvinisten zu überzeugen:

C'est quelque chose de bien triste d'être réduits à la nécessité de chercher chez les princes luthériens faute d'en trouver chez les réformés, car il n'est que trop vrai qu'il se trouve bien de difficultés à établir notre peuple en Irlande, en Brandebourg et dans le pays de HesseCassel. [...] Il ne faut pas espérer qu'on fasse en Brandebourg aucune dépense pour y établir des réfugiés et d'ailleurs il m'a paru que Mr. de Barfuss s'accommoderait mieux des Silésiens qui demandent d'être reçus en ce pays là que de François qui souhaitent de s'y établir ${ }^{24}$.

Trotz zahlreicher Bittschriften de Mirmands und von Dohnas blieben die Grenzen Brandenburg-Preußens weiteren Refugiés zunächst verschlossen ${ }^{25}$. Erst 1699 ließ sich der Kurfürst davon überzeugen, den in der Schweiz unerwünschten französischen Flüchtlingen die gleichen Privilegien wie den nach 1685 nach Brandenburg-Preußen gekommenen Refugiés zu gewähren. Daß dabei im Vorfeld ein gewisser Druck von Seiten der hugenottischen Botschafter am Hof der Hohenzollern ausgegangen war, läßt sich am Text seiner Résolution ablesen:

Sa Sérénité Electorale de Brandebourg, ayant suffisamment entendu la très respectueuse exposition que les François Refugiés dans le Canton de Berne en Suisse lui ont fait faire par

Preußen, Irland und Großbritannien: ein Vergleich, in: Manuela BOHM, Jens HÄSELER, Robert VIOLET (Hg.), Hugenotten zwischen Migration und Integration. Neue Forschungen zum Refuge in Berlin und Brandenburg, Berlin 2005, S. 37-58; DIES., Huguenots in Ireland, Britain and Brandenburg-Prussia (1660-1750), in: DIES. (Hg.), Religious Refugees in Europe, Asia and North America, Hamburg 2006 (Atlantic Cultural Studies, 4).

${ }^{22}$ MYLIUS, Recueil (wie Anm. 16), Sp. 65-72.

${ }^{23}$ BibSHPF (wie Anm. 2), Papiers Court, Nr. 17 M, M 617.

${ }^{24}$ Ibid., Ms. 618 AA.

${ }^{25}$ Ibid., Copies, Ms. 618 AA. 
leurs Députés [... beschließt] de prendre \& recevoir les dits François Refugiés [...] sous sa haute protection, comme les autres Réfugies receus \& établis dans ses Estats ${ }^{26}$.

Ein gewisser Widerwillen des Kurfürsten ist weiterhin erkennbar, wenn es heißt:

Quoi que Sa Sérénité Electorale ait déjà fait une dépense considérable pour l'établissement et l'entretien d'un grand nombre de Refugiés de France, du Palatinat, \& du Piémont; cependant Elle a voulu encore sur la très humble demande des dits Députés à ce sujet, donner suivant sa promesse la présente Résolution ${ }^{27}$.

Der hartnäckigen Arbeit der Députés war also nach Jahren der Zurückhaltung des Kurfürsten in Sachen weiterer Ansiedlung erneut Erfolg beschieden. Doch auch wenn im Text explizit den Neuankömmlingen die gleichen Privilegien gewährt wurden wie 1685 im Edikt von Potsdam, so enthält die Résolution einige Zusätze, die die Probleme mit den 14 Jahre zuvor ins Land gekommenen Flüchtlingen deutlich reflektieren: enttäuschte Erwartungen der Regierung bezüglich der Wirtschaftskraft der Neubürger, Zusammenstöße zwischen deutschen und französischen Untertanen und Institutionen und Ähnliches. 1699 manifestierte sich trotz der Garantie der Sonderstellung der Refugiés nicht nur der Widerwillen der Regierung gegen die weitere Ansiedlung, sondern es wird, und dies ist neu gegenüber dem Edikt von Potsdam, explizit die Unterordnung der Refugiés unter die Gesetze des Landes verlangt: Nicht nur werden die Refugiés nicht mehr als bien Receus ${ }^{28}$ bezeichnet, sondern les impétrants seront obligés en arrivant de se conformer aux Loix \& à la Police du Pais, \& de s'engager par le serment de fidélité, \& de soumission ${ }^{29}$. Hier scheinen sich bereits erste Anzeichen der Intention der Regierung, die Neuankömmlinge zu einer gewissen primären Integration avant la lettre zu zwingen, bemerkbar zu machen $^{30}$.

Als 1703 Ludwig XIV. Frankreich das Fürstentum Orange einverleibte und die dort lebenden Kalvinisten ultimativ aufforderte, entweder zum Katholizismus zu konvertieren oder aber auszuwandern, wurde die FranzösischReformierte Kirche Berlins dann in Sachen Orangeois $^{31}$ erneut aktiv. Sie versuchte die Hohenzollern, zunächst über den Mittelsmann von Dohna, für die

${ }^{26}$ MYLIUS, Recueil (wie Anm. 16), Sp. 129.

${ }^{27}$ Ibid., Sp. 129-134.

${ }^{28}$ Edikt von Potsdam wie abgedruckt in MYLIUS, Recueil (wie Anm. 16), Sp. 44.

${ }^{29}$ Ibid., Sp. 129.

${ }^{30}$ Unter »primärer Integration « wird hier für den Bereich »Recht und Ordnung« die Akzeptanz des und die Unterordnung unter das Recht des Aufnahmelandes verstanden. In BrandenburgPreußen ging dies nicht mit der Aufgabe des eigenen, französischen Rechts einher, sondern führte zu einer Koexistenz von landespezifischem Recht und französischem Recht.

${ }^{31}$ Vgl. hierzu den Artikel von Françoise Moreil in diesem Band. 
Aufnahme der Orangeois zu gewinnen ${ }^{32}$. Doch erst 1709 bestätigte König Friedrich I. nicht nur das Edikt von Potsdam, sondern naturalisierte gleichzeitig alle französischen Protestanten in Brandenburg-Preußen, einschließlich all derer, die sich nach 1709 noch ansiedeln würden ${ }^{33}$. De iure hätte eine Naturalisierung eigentlich eine rechtliche Gleichstellung mit den deutschen Untertanen des Landes nach sich ziehen müssen. Die Refugiés behielten jedoch ihre rechtliche und wirtschaftliche Sonder- und Besserstellung bei. Darüber hinaus wurden ihnen nun auch die Privilegien deutscher Untertanen zugestanden ${ }^{34}$.

König Friedrich Wilhelm I. versuchte diesem $>$ Staat im Staat 1715 ein Ende zu setzen und die Refugiés mit den deutschen Untertanen gleichzustellen: Im Kombinationsedikt wurde die Abschaffung des Ministers für französische Angelegenheiten, die Übernahme aller Colonie-Angelegenheiten durch das Generalkriegskommissariat, die Eingliederung der hohen französischen Gerichtsinstanzen in das Geheime Justiz-Collegium sowie der französischen Untergerichte in die deutschen Magistrate verfügt ${ }^{35}$. Auch in Kirchensachen war eine Vereinheitlichung intendiert. 1717 sollte auch für die Französisch-Reformierte Kirche der Heidelberger Katechismus in französischer Übersetzung eingeführt und damit ein gewisser Konformismus unter den Reformierten BrandenburgPreußens etabliert werden ${ }^{36}$. Diese Pläne scheiterten jedoch am Widerstand der Colonie. Stattdessen wurde 1718 das sogenannte Oberdirektorium (Grand Directoire oder Conseil français) eingerichtet, das für alle Anliegen der Kolonisten und die Förderung der Manufakturen zuständig war. Auffallend ist die große Zahl von Refugiés in der 'Direktionı der Colonien. Zwar oblag die Leitung der obersten Behörde in der Regel einem Deutschen, doch waren die Refugiés in Brandenburg-Preußen sehr viel mehr als in Hessen-Kassel an Verwaltung und Aufsicht der französischen Colonien beteiligt.

1720 wurden die Privilegien von 1685, aber auch die Androhung von Strafen beim Verlassen des Landes ohne Erlaubnis des Königs ${ }^{37}$, erneut bestätigt. Zur Vereinheitlichung des Rechts für Immigranten bzw. andere in Berlin lebende Nationen wurde allen neuen Immigranten, egal welcher Nationalität und Religion, die Möglichkeit gegeben, sich entweder als Stadtbürger oder als Colonie-Bürger registrieren zu lassen ${ }^{38}$. So kamen Schweizer, französische Katholiken und andere in den Genuß der Privilegien der Colonie. Während in

32 AFrD (wie Anm. 14), Registre des Actes consistoriaux de l'Eglise française reformée de Berlin, Bd. 1, 10. Juni 1672-4. Juli 1694, fol. 145r, 150r und 160r.

${ }^{33}$ MYLIUS, Recueil (wie Anm. 16), Sp. 257-260.

${ }^{34}$ Vgl. Edouard MURET, Geschichte der Französischen Kolonie in Brandenburg-Preußen, unter besonderer Berücksichtigung der Berliner Gemeinde, Berlin 1885, S. 307; Helmut ERBE, Die Hugenotten in Deutschland, Essen 1937, S. $57 \mathrm{f}$.

${ }^{35}$ BRANDENBURG, Rechtsprechung (wie Anm. 19), S. 296.

${ }^{36}$ MYLIUS, Recueil (wie Anm. 16), Sp. 515-518.

${ }^{37}$ JERSCH-WENZEL, Juden (wie Anm. 13), S. 103-106.

${ }^{38}$ MYLIUS, Recueil (wie Anm. 16), Sp. 557-562 und vor allem Sp. 567-572. 
rechtlicher Hinsicht die Kolonisten bereits 1742 dem preußischen Kriminalrecht unterworfen und 1794 die Ordonnance française abgeschafft wurde, d.h. alle Kolonisten bzw. ihre Gerichte nun dem Allgemeinen Preußischen Landrecht unterworfen waren, wurden der Colonie-Status und damit die meisten Sonderrechte und Institutionen - bis auf die kirchlichen - der Colonie erst 1809 aufgehoben ${ }^{39}$.

\section{Zusammenfassung}

Der Vergleich der Einwanderungspolitik Hessen-Kassels mit der Brandenburg-Preußens zeigt, daß die Privilegien in Brandenburg-Preußen umfassender waren und blieben, allerdings mit der wichtigen Einschränkung, daß Freizügigkeit bzw. der Abzug vor allem von französischen Manufakturisten und Gewerbetreibenden in Brandenburg-Preußen ohne die explizite Erlaubnis des Landesherren verboten waren. Die Privilegien hatten ihren Preis. Mit der privilegierenden Immigrationspolitik war kein Bleiberecht, sondern für bestimmte Gruppen ein Bleibezwang verbunden.

Auch wenn beide Landesherren, Landgraf Karl und Kurfürst Friedrich Wilhelm, 1685 bereit waren, im Fall der Hugenotten kulturelle Diversität in ihren Staaten nicht nur zu akzeptieren, sondern zu fördern, so war Hessen-Kassel von Anfang an wesentlich mehr als Brandenburg-Preußen auf eine wirtschaftliche und rechtliche Gleichstellung der Immigranten bedacht und zeigte bereits 1685 ein geringeres Interesse an der Ansiedlung von französisch-reformierten Protestanten. Eine rechtliche und wirtschaftliche Gleichstellung von Refugiés und deutschen Untertanen mit dem besonderen Augenmerk auf die Achtung von Ordnung und Gesetzen des Landes, d.h. die Integration der Immigranten in diesen Bereichen, wie sie sich in Hessen-Kassel schon in der Regierungszeit Landgraf Karls als langfristiges Ziel andeutete, wurde in BrandenburgPreußen nur kurzfristig 1699 angedacht bzw. unter König Friedrich Wilhelm I. (1688-1740, König seit 1713) in Angriff genommen. Die Hürden, die in Brandenburg-Preußen zu nehmen waren, waren ungleich höher, denn nicht nur die Sonderjurisdiktion, sondern letztendlich auch das "fremde Recht" im Land, sprich das 1685 für die Colonien etablierte französische Recht, hätten abgeschafft werden müssen. Auch der Versuch König Friedrich Wilhelms I., dem Beispiel Englands folgend, mit der Zurücknahme der Sonderrechte der französischen Immigranten auch mehr Konformismus in Religionssachen durchzusetzen, scheiterte 1715 am Widerstand der Refugiés. Der Sonderweg

${ }^{39}$ Siehe MURET, Kolonie (wie Anm. 34), S. 307-310 und BRANDENBURg (wie Anm. 19), S. $296 f$. 
Brandenburg-Preußens - nicht nur im Vergleich mit anderen deutschen Staaten, sondern im gesamteuropäischen Vergleich - zeigt sich auch deutlich darin, daß die Vereinheitlichung des rechtlichen Status aller Untertanen für Immigranten bzw. Angehörige anderer »Nationen « die Alternative bot, Teil der französischen Colonie zu werden oder sich unter das jeweilige deutsche Recht zu begeben. Diese Koexistenz von französischem und deutschem Rechtssystem wurde erst 1794 durch das Allgemeine Preußische Landrecht aufgehoben.

Die Akzeptanz von kultureller Diversität in Hessen-Kassel und Brandenburg-Preußen hatte nicht nur zur Folge, daß auf dieser Ebene von Seiten der Regierungen bis nach 1750 kaum Versuche unternommen wurden, die Immigranten kulturell oder sozial zu integrieren. In Brandenburg-Preußen kopierten adlige und bürgerliche Eliten vielmehr die »Kultur" - oder was sie dafür hielten - des französisch-reformierten Adels bzw. der bürgerlichen Eliten ${ }^{40}$.

Auffallend für beide Aufnahmeländer ist der beeindruckende Einfluß, den die Refugiés auf die Gestaltung von Immigrations- und Ansiedlungspolitik zu nehmen wußten. Für Brandenburg-Preußen ist hier trotz der Versuche Friedrich Wilhelms I., die 1685 gewährten Privilegien zu beschneiden, der größere und kontinuierlichere Erfolg der Refugiés zu verzeichnen.

\section{Résumé français}

Tandis que le landgraviat de Hesse-Cassel accueillait des réfugiés religieux réformés notamment pour des raisons économiques, l'édit de Potsdam se présenta comme un édit d'accueil et de tolérance qui garantissait des privilèges extraordinaires aux huguenots dans la diaspora allemande.

Non seulement dans le Brandebourg, mais aussi dans le cas de la Hesse-Cassel, les huguenots réfugiés cherchaient à prendre de l'influence sur les privilèges qui devaient leur être accordés. Très peu de travaux concernant ce sujet considèrent le rôle actif que les réfugiés jouaient dans les négociations avec les princes européens. Peu de chercheurs tiennent compte des sources témoignant de l'aversion croissante du landgrave (à partir de 1688) et de l'électeur (depuis 1692) d'accueillir encore plus de réfugiés après 1690 et de garantir les privilèges de 1685 . Il semble que les princes espéraient plus des réfugiés que ces derniers ne pouvaient leur apporter. Par conséquence, l'afflux des huguenots devait selon eux être stoppé.

Établis comme une nation à part, non seulement dans le Brandebourg, mais aussi en Hesse-Cassel, l'intégration avant la lettre devint un objectif important, en Brandebourg, sous le roi Frédéric-Guillaume, en Hesse-Cassel, sous les successeurs du landgrave Charles. Ces premières tentatives d'intégrer les réfugiés déjà vers la fin du XVII ${ }^{e}$ et au début du $\mathrm{XVIII}^{\mathfrak{c}}$ siècle échouèrent. Dans ces deux états allemands, la nation française conserva ses privilèges au moins jusqu'au début du $\mathrm{XIX}^{e}$ siècle.

${ }^{40} \mathrm{Vgl}$. hierzu ausführlich den Aufsatz von Jens Häseler in diesem Band. 
